\title{
Effective field theory approach to fluctuation-induced forces between colloids at an interface
}

\author{
Cem Yolcu, Ira Z. Rothstein, and Markus Deserno \\ Department of Physics, Carnegie Mellon University, 5000 Forbes Ave., Pittsburgh, Pennsylvania 15213, USA
}

(Received 26 July 2010; revised manuscript received 1 December 2011; published 24 January 2012)

\begin{abstract}
We discuss an effective field theory (EFT) approach to the computation of fluctuation-induced interactions between particles bound to a thermally fluctuating fluid surface controlled by surface tension. By describing particles as points, EFT avoids computing functional integrals subject to difficult constraints. Still, all information pertaining to particle size and shape is systematically restored by amending the surface Hamiltonian with a derivative expansion. The free energy is obtained as a cumulant expansion, for which straightforward techniques exist. We derive a complete description for rigid axisymmetric objects, which allows us to develop a full asymptotic expansion - in powers of the inverse distance-for the pair interaction. We also demonstrate by a few examples the efficiency with which multibody interactions can be computed. Moreover, although the main advantage of the EFT approach lies in explicit computation, we discuss how one can infer certain features of cases involving flexible or anisotropic objects. The EFT description also permits a systematic computation of ground-state surface-mediated interactions, which we illustrate with a few examples.
\end{abstract}

DOI: 10.1103/PhysRevE.85.011140

PACS number(s): 05.40.-a, 03.50.-z, 68.05.-n

\section{INTRODUCTION}

Be it the electromagnetic field, the curvature of a surface, or the composition of an inhomogeneous mixture, boundaries or objects interacting with a field generally place constraints on the fluctuations of it. This is known to lead to interactions between the constraining objects. The earliest and most famous example of this effect was discussed in 1948 by Casimir, who showed that two charge-neutral conducting plates in vacuum attract due to the constraints they impose on the quantum fluctuations of the electromagnetic field [1-3]. Today it has become customary to name such interactions after Casimir, even in cases where the underlying fluctuations are not quantum mechanical but thermal in origin [4-6]. In this article, we will investigate a particular incarnation of this effect, namely, forces induced by thermal fluctuations between particles bound to a fluid surface characterized by surface tension [7-13].

The nontrivial aspects of such calculations tend to arise from the constraints that the extended objects impose on the partition sum. This issue is usually dealt with by pinning the field to the surface of the objects through delta functions in the integration measure [10]. A clear exposition of this method, applied to compact objects in fluid membranes and films, can be found in Ref. [11]. This method was later refined in the context of the electromagnetic Casimir effect [14]. In the latter work, the constraints of the objects enter the interaction energy through their scattering matrix coefficients. In fact, usage of scattering approaches for electromagnetic Casimir interactions has a long history and has led to recent developments [15-18] in the field, concerning analytical results. Very recently, thermal Casimir interactions between biological membrane inclusions were studied in a similar spirit [19].

In this paper we employ a different strategy to streamline the boundary condition issue, namely, reverting to a point-particle description. We emphasize right from the start that this is not an uncontrolled approximation for the shape of the extended objects, because we can systematically rescue the complete finitesize information by means of effective field theory (EFT). The key philosophy behind EFT is separation of scales, which is deeply rooted in the concept of renormalization (for a review, see Ref. [20]). Originally developed in the context of quantum field theory, it has been applied only quite recently in a purely classical framework by Goldberger et al. [21], who used it to study the gravity wave profile for in-spiraling black holes. This particular incarnation of EFT has subsequently been utilized to derive not only new results in gravitational wave physics [22] but also to calculate the leading-order finite-size correction to the Abraham-Dirac-Lorentz radiation reaction force law in classical electrodynamics [23]. Both of these applications dealt with intricate boundary condition problems for classical nonfluctuating fields, whereas the present article extends the formalism to allow for finite temperatures. We recently introduced this generalization in Ref. [13]; here we develop the formalism in greater detail, expand on our previous results, and discuss further physical situations.

Examples of point-particle approaches employed to compute surface-mediated forces have appeared several times [24-26] in the context of biological membranes. However, in most of these references the final answer depends on the short wavelength cutoff of the continuum theory, which is ambiguous by construction. To alleviate the issue, the cutoff is usually fixed by reinterpreting it as a multiple of the inclusion size, but there are at least two problems with this: (1) The size of the objects embedded in the field and the short wavelength cutoff of the theory are generally unrelated, and (2) even if the objects do have sizes of the same order as the cutoff, it is not clear how to treat particles with different radii. Instead, the proper way to free physical quantities from dependence on some ambiguous cutoff is via renormalization techniques. Our approach is constructed such that the cutoff never "contaminates" the interactions and therefore never inappropriately restricts particle sizes. Another difference between earlier point-particle approaches and ours is the fact that our treatment is not approximate in that all finite-size information is systematically retained in the point-particle picture. Unless this is done, it would not even make sense to attempt calculating higher-order corrections to asymptotically correct results. 
For the sake of clarity, we restrict this paper to the case of rigid particles, e.g., colloids adsorbed at a fluid-fluid interface. In other words, within the region of the surface occupied by the particle, fluctuations are not only reduced, but are frozen, in a manner compatible with the boundary conditions at the circumference of the particle and its permissible rigid body motions. Furthermore, we will assume the shapes of the particles are such that they have a circular footprint on the surface. While these two assumptions allow us to carry out a more transparent discussion of the EFT formalism, we nonetheless outline what is entailed in relaxing these assumptions. We also briefly turn our attention to interactions that are induced not by fluctuations but by permanent shape irregularities of the particles. The formalism allows a systematic approach to calculate these interactions without resorting to a superposition assumption to disentangle the boundary conditions of the particles from one another.

In the following section, we begin our discussion by reviewing the nature of the surface and fluctuation-induced interactions we are interested in, as well as outlining our effective field theory approach. We then proceed in Sec. III with constructing the effective theory and computing interactions in an asymptotic expansion. First, we illustrate the computation by considering only one of the many terms in the expansion as an example in Sec. III B. Then, we derive the interaction free energy of a pair of particles as a full expansion, i.e., valid at arbitrary separation, and extract the leading near-contact behavior. We then compute the leading nontrivial triplet and quadruplet interactions, as well as briefly discuss the case of soft or nonaxisymmetric particles. Finally, we show how the formalism can be used for problems where the particles impart a permanent deformation on the surface. To separate the main arguments from mundane technicalities, we have collected details of certain calculations as well as auxiliary discussions in four appendices.

\section{FORMALISM}

\section{A. The surface}

We are interested in a fluid surface whose behavior is determined by the surface tension energy $\sigma \int d A$, where the integral encompasses the entire surface and $\sigma$ is the surface tension. A nearly flat surface can be parameterized using the Monge gauge [27], describing the shape in terms of its orthogonal deviation $h$ from a flat $x y$ base plane. This yields the energy functional

$$
\mathcal{H}_{\text {surf }}[h]=\sigma \int_{\mathcal{S}} d^{2} r \sqrt{1+h_{i}^{2}(\boldsymbol{r})},
$$

where we write the gradient with the partial derivative subscript $i$. Note that the domain $\mathcal{S}$ of the integral is not the whole $\mathbb{R}^{2}$ but excludes the regions defined by the projection of the colloids onto the base plane. The presence of the particles imposes conditions on $h$ at the boundaries of these regions. For concreteness, let us picture circular disks floating on the surface, which dictate the height of the fluid film anywhere along their circumference.

Under the further assumption of a weakly deformed surface, $|\nabla h| \ll 1$, one finally arrives at the quadratic surface
Hamiltonian

$$
\mathcal{H}_{\text {surf }}[h]=\frac{1}{2} \sigma \int_{\mathcal{S}} d^{2} r h_{i}^{2}(\boldsymbol{r}),
$$

up to an irrelevant constant. The existence of this integral defines the function space that $h(\boldsymbol{r})$ belongs to.

\section{B. Fluctuation-induced interactions}

At a given temperature, the surface will fluctuate around its ground-state shape, which minimizes the energy as defined in Eq. (2), while still subject to the boundary conditions imposed by the particles, i.e., the continuity of the surface along their circumference. These local constraints on the fluctuations will result in the partition function and the free energy being dependent on the spatial arrangement of the constrained regions, hence giving rise to forces.

Formally, the free energy is given as

$$
\beta F=-\log \int \mathcal{D} h \mathrm{e}^{-\beta \mathcal{H}_{\text {surf }}[h]},
$$

where the partition function is a functional integral over all permissible field configurations $h(\boldsymbol{r})$, i.e., those compatible with the boundary conditions imposed by the particles. This integral is a Gaussian integral, on account of the Hamiltonian (2) being quadratic in the field, but its evaluation is not straightforward. Though one can formally write the free energy (3) as $\sim \log \operatorname{det} \mathbb{K}$, this does not help with explicit computation, as the kernel $\mathbb{K}=-\nabla^{2}$ [see Eqs. (2) and (3)] is hard to diagonalize in this constrained function space. ${ }^{1}$ One wishes to free the functional integral in Eq. (3) from the finite-sized regions of constraint, such that the integral becomes a "straightforward" Gaussian.

One possible means to this end involves extending the validity of the Hamiltonian (2) to the entirety of $\mathbb{R}^{2}$ at the expense of modifying the integration measure by delta functions, appropriately chosen to make sure the functional integral in Eq. (3) sifts out only permissible field shapes $h(\boldsymbol{r})[7-11,14,24,28]$. In this article we follow another path, which encodes the constraints in additional terms to the free-surface Hamiltonian. This is different from the approach taken in Refs. [28,29] in that it is not necessary to assume these additional terms to be small, which corresponds to the case when the constraints on the fluctuation of the particles are not rigid. Even when one deals with rigid constraints, one can compute the free energy in a convergent perturbation expansion. This is achieved by means of an effective field theory (EFT).

\footnotetext{
${ }^{1}$ The integration kernel in Eq. (2) involves a Laplacian operator that acts on functions defined only over a subset $\mathcal{S}$ of $\mathbb{R}^{2}$. Integrating by parts, Eq. (2) can be massaged into $\frac{\sigma}{2} \int_{\mathcal{S}} d^{2} r h\left(-\nabla^{2}\right) h+\frac{\sigma}{2} \int_{\partial \mathcal{S}} d \ell \hat{n}_{i} h \partial_{i} h$, which does not have the form $\frac{1}{2} \int d^{2} r d^{2} r^{\prime} h(\boldsymbol{r}) K\left(\boldsymbol{r}, \boldsymbol{r}^{\prime}\right) h\left(\boldsymbol{r}^{\prime}\right)$ with a translationally invariant kernel $K\left(\boldsymbol{r}, \boldsymbol{r}^{\prime}\right)=K\left(\boldsymbol{r}-\boldsymbol{r}^{\prime}\right)$, which could be diagonalized easily in momentum space.
} 


\section{The effective theory}

As we have alluded to before, we want to describe the system with an effective Hamiltonian such that we can write the partition function as $Z=\int \mathcal{D} h \mathrm{e}^{-\beta \mathcal{H}_{\text {eff }}[h]}$ with the field variations unconstrained. This allows the evaluation of the integral over a Gaussian measure with the familiar translationally invariant harmonic kernel. To this end, we construct the effective theory as $\mathcal{H}_{\text {eff }}=\mathcal{H}+\Delta \mathcal{H}$ where $\mathcal{H}$ is the free surface Hamiltonian

$$
\mathcal{H}[h]=\frac{1}{2} \sigma \int_{\mathbb{R}^{2}} d^{2} r h_{i}^{2}(\boldsymbol{r}),
$$

and the perturbation $\Delta \mathcal{H}$ is a collection of local terms specifically designed to capture the constraints, as we will discuss shortly. In this form, the effective theory describes a system of pointlike particles embedded in an otherwise homogeneous surface.

The free energy, measured with respect to the free energy of the unperturbed surface, is then calculated in a cumulant expansion

$$
\begin{aligned}
\beta F & =-\log \int \mathcal{D} h \mathrm{e}^{-\beta \mathcal{H}} \mathrm{e}^{-\beta \Delta \mathcal{H}} \\
& =-\log \left\langle\mathrm{e}^{-\beta \Delta \mathcal{H}}\right\rangle=-\sum_{q=1}^{\infty} \frac{1}{q !}\left\langle(-\beta \Delta \mathcal{H})^{q}\right\rangle_{\mathrm{c}} .
\end{aligned}
$$

The last step above can be taken as the definition of the cumulants denoted as $\langle\ldots\rangle_{\mathrm{c}}$, which involve many-point connected correlation functions of the field. Since averages are computed in the Gaussian ensemble determined by the free Hamiltonian $\mathcal{H}$, the many-point correlators break up into a product of two-point correlators

$$
\left\langle h(\boldsymbol{r}) h\left(\boldsymbol{r}^{\prime}\right)\right\rangle=\frac{1}{\beta \sigma} G\left(\boldsymbol{r}, \boldsymbol{r}^{\prime}\right)=-\frac{1}{4 \pi \beta \sigma} \log \left(\boldsymbol{r}-\boldsymbol{r}^{\prime}\right)^{2},
$$

due to Wick's theorem. Here $G\left(\boldsymbol{r}, \boldsymbol{r}^{\prime}\right)$ is the Green function or inverse of operator $-\nabla^{2}$, i.e., $-\nabla^{2} G\left(\boldsymbol{r}, \boldsymbol{r}^{\prime}\right)=\delta\left(\boldsymbol{r}-\boldsymbol{r}^{\prime}\right)$.

The aim is that the effective theory reproduces the correct free energy: that of the original system with finite-sized regions of constraint, or the full theory as we will henceforth refer to it. This is ensured by matching a set of physical observables in the two theories, a procedure that will be explored in detail further in Sec. III A.

In general, $\Delta \mathcal{H}$ consists of all polynomials in the derivatives of the field $h$ consistent with the symmetries of the problem, evaluated at the position of each particle. As such, it has the form of an operator product expansion [30] involving terms like $A_{a} h_{i}^{2}\left(\boldsymbol{r}_{a}\right), B_{a} h_{i j}^{2}\left(\boldsymbol{r}_{a}\right), C_{a} h_{i j}^{2}\left(\boldsymbol{r}_{a}\right) h_{i}^{2}\left(\boldsymbol{r}_{a}\right)$, etc., where the label $a$ denotes a particle and $\boldsymbol{r}_{a}$ its appropriately defined position, e.g., the center of a circular disk. The prefactors $A, B$, $C, \ldots$ are called Wilson coefficients and have to be fixed by the aforementioned matching procedure. An expansion of similar spirit was employed in Ref. [31] for studying interactions involving spherical objects in a critical fluid.

Let us briefly discuss the temperature dependence of the free-energy expansion (6). Every cumulant, being the thermal average of a power of $\beta \Delta \mathcal{H}$, will involve many-point correlators that consist of a multiplicative combination of terms in $\Delta \mathcal{H}$. Recalling from Eq. (7) that every pair of field occurrences in these many-point correlators carries a factor $\beta^{-1}=k_{\mathrm{B}} T$, each quadratic term in $\beta \Delta \mathcal{H}$ can be counted as a factor $\beta^{0}$, each quartic term as $\beta^{-1}$, and so on, in the term of expansion (6) they appear in. From this we see that the traditionally encountered form $F=k_{\mathrm{B}} T f\left(\left\{\boldsymbol{r}_{a}\right\}\right)$ of fluctuation-induced interactions [7-11,24,28,29] stems from quadratic terms in $\Delta \mathcal{H}$. This allows the interpretation of the interactions as those between induced "capillary charges," arising from thermal fluctuations around each particle and subsequent polarizations due to the deformation that propagates from those. Similarly, one can see that higher-than-quadratic terms in $\Delta \mathcal{H}$ give rise to an excess of factors of $k_{\mathrm{B}} T$ in the cumulants in Eq. (6). Therefore, such terms in the effective theory produce a free energy $F \sim\left(k_{\mathrm{B}} T\right)^{p \geqslant 2}$. These are higher-order fluctuation corrections to the interaction. However, to treat these nonlinear corrections properly, one has to also consistently relax the weak deformation assumption on the free-surface Hamiltonian (2). This involves quartic (or higher-order) terms in the free Hamiltonian that can also be treated perturbatively. While such extensions do not affect the basic EFT formalism, they require additional field theoretical sophistication that would distract from the basic idea we wish to communicate here, and hence we defer such nonlinear corrections to future work.

\section{EFT OF FLAT DISKS ON A FILM}

After briefly outlining the main idea of our formalism, we will now proceed by applying it to a specific problem. We want to compute the fluctuation-induced interaction between colloids with a circular footprint on the base plane. For this, we write the effective theory as $\Delta \mathcal{H}=\sum_{a} \Delta \mathcal{H}_{a}$, where $a$ labels the particles. For one particle located at $\boldsymbol{r}_{a}, \Delta \mathcal{H}_{a}$ is a derivative expansion of the form

$$
\Delta \mathcal{H}_{a}=\left.\frac{1}{2}\left(C_{a}^{(0)} h^{2}+C_{a}^{(1)} h_{i}^{2}+C_{a}^{(2)} h_{i j}^{2}+\cdots\right)\right|_{\boldsymbol{r}=\boldsymbol{r}_{a}},
$$

encoding a quadratic excess energy for deforming the field in the vicinity of that particle. Denoting the radius of disk $a$ by $R_{a}$, dimensional analysis shows that each Wilson coefficient $C_{a}^{(\ell)}$ scales as $\sigma R_{a}^{2 \ell}$, such that the limit $R_{a} \rightarrow 0$ is well defined. It is also worth pointing out that, applying a Hubbard-Stratonovich transformation to the partition function in Eq. (5), the effective Hamiltonian $\mathcal{H}_{\text {eff }}=\mathcal{H}+\sum_{a} \Delta \mathcal{H}_{a}$ can be brought into the form of the effective action of Emig et al. [14]

As we mentioned earlier, the terms in Eq. (8) represent induced charges localized at the points $\boldsymbol{r}_{a}$. Thus, the coefficients $C_{a}^{(\ell)}$ will be referred to as polarizabilities. One can see this clearly by considering a generic term in Eq. (8) around an applied or background field. Choosing $\boldsymbol{r}_{a}=0$ and dropping the label $a$ for convenience, one substitutes $h \rightarrow h^{\text {bg }}+h$ into the term $(1 / 2) C^{(\ell)}\left(\partial^{\ell} h\right)^{2}$ and observes the term linear in $h$ :

$$
C^{(\ell)} \partial^{\ell} h^{\mathrm{bg}}(0) \partial^{\ell} h(0)=\int d^{2} r h(\boldsymbol{r}) C^{(\ell)}(-\partial)^{\ell}\left[\delta(\boldsymbol{r}) \partial^{\ell} h^{\mathrm{bg}}(0)\right] .
$$

After integrating by parts, one can identify the localized source:

$$
J(\boldsymbol{r})=(-)^{\ell} C^{(\ell)} \partial^{\ell}\left[\delta(\boldsymbol{r}) \partial^{\ell} h^{\mathrm{bg}}(0)\right]
$$




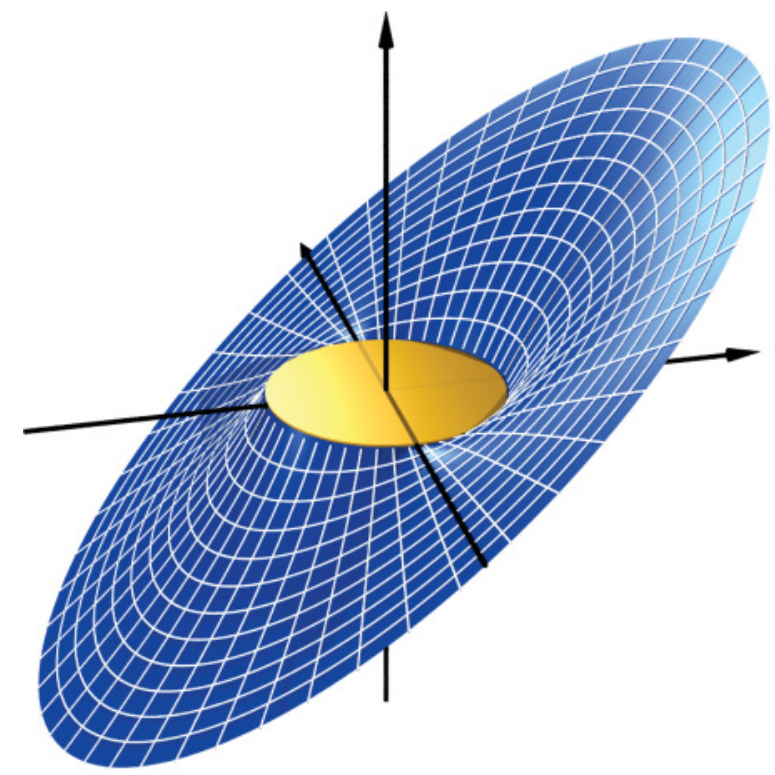

FIG. 1. (Color online) The figure illustrates how a rigid, horizontally fixed particle of radius $R$ creates a local field deformation when exposed to a tilted planar background, $h^{\text {bg }}=r \cos \varphi$. The total deformation is the sum of the background and the induced deformation, $\delta h=-\left(R^{2} / r\right) \cos \varphi$. The discontinuity of the (normal) gradient of the total shape along the circumference of the particle is a dipole-like $(\sim \cos \varphi)$ "capillary polarization" that can be thought of as sourcing the response $\delta h$.

This source is an $\ell$ th-order multipole induced by the $\ell$ th derivative of the background, created by thermal fluctuations or the field of another such charge.

The constraints imposed on the motion of the particle (typically by the experimental situation at hand) determine whether a certain multipole charge can be induced or not. For instance, if the particle is free to move vertically, there will be no monopole induced, or $C^{(0)}=0$, because the particle can accommodate a nonzero $h(0)$ by moving up or down, without an excess energy cost. Similarly, the dipole polarizability $C^{(1)}$ vanishes if there are no constraints on the tilt degree of freedom of the particle, as it can then adjust freely to a nonzero $\partial_{i} h(0)$. We will reprise these arguments in due time when we discuss the leading-order interaction between the particles.

The presence of a multipole like Eq. (10) on the surface creates a deformation around it, which is simply the following convolution of the charge with the Green function:

$$
\begin{aligned}
\delta h(\boldsymbol{r}) & =-\frac{1}{\sigma} \int d^{2} r^{\prime} J\left(\boldsymbol{r}^{\prime}\right) G\left(\boldsymbol{r}^{\prime}, \boldsymbol{r}\right) \\
& =-\frac{C^{(\ell)}}{\sigma} \partial^{\ell} h^{\mathrm{bg}}(0) \partial^{\ell} G(0, \boldsymbol{r}) .
\end{aligned}
$$

For example, Fig. 1 depicts the response of a horizontally fixed disk to a dipole background, $h^{\mathrm{bg}}=r \cos \varphi$. The fluctuationinduced interaction can be viewed as the interaction between these induced multipoles mediated by the surface.

Notice that the multipole responses of order $\ell>0$ decay as $r^{-\ell}$, whereas the monopole response does not. In fact, the monopole response is not even square integrable, i.e., its energy Eq. (2) diverges logarithmically. However, this is not a property of the EFT point-particle description, but of the surface Hamiltonian itself. Therefore, calculating interactions between objects with nontrivial monopole responses in $\mathbb{R}^{2}$,i.e., disks with pinned height, is intrinsically an ill-defined problem and requires regularization of the surface Hamiltonianwhether one is using EFT or not. The issue can be alleviated by considering a finite subset of $\mathbb{R}^{2}$ or modifying the theory with a damping term. However, since this would distract from the main idea we want to communicate in this article, we will defer this case to Appendix D and avoid monopoles in the main text by restricting to particles that can fluctuate up and down freely, i.e., particles for which $C^{(0)}=0$.

Having discussed the multipole responses, one more note for completeness is in order. Although one must build $\Delta \mathcal{H}$ from all scalars that the symmetries of the problem allow, terms involving repeated indices, such as $h_{i i}^{2}, h_{i i j}^{2}$, etc., are redundant. One way to see this is that the response Eq. (11) of such a charge to a background would necessarily be local, as $G_{i i}(0, \boldsymbol{r}) \equiv \delta(\boldsymbol{r})$, and hence such charges do not interact with anything away from them.

\section{A. Fixing the polarizabilities: Matching}

Equation (8) is the most general quadratic Hamiltonian compatible with the circular symmetry of one particle. The only thing we have not established yet are the exact values of the polarizability coefficients, which we will attend to in this section. These values are fixed by matching a set of observables in the effective (point-particle) and full (finite-sized particle) theories. Although any suitably chosen set of observables will do, we will consider the most obvious one here: the polarization response to an applied field. If one is dealing with more complicated physics and boundary conditions or shapes, a different choice of observable may be more practical (e.g., for numerical matching) or even necessary.

We gave the general expression for an $\ell$ th-order induced multipole response in Eq. (11). Clearly, the background that will induce this response is one that is $\ell$ th order in the coordinates, and the deformation decays like $\partial^{\ell} G(0, \boldsymbol{r}) \sim r^{-\ell}$. The polarizability $C^{(\ell)}$ should be fixed such that this EFT response is identical to the response in the full theory of the particle to the same background. Given the correspondence between our present Hamiltonian and classical electrostatics in two dimensions, the latter response is found analogously to the problem of a conductor in an external electric field.

If we write the derivatives in Eq. (11) explicitly, this expression involves the index contraction $h_{i_{1} \cdots i_{\ell}}^{\mathrm{bg}}(0) G_{i_{1} \cdots i_{\ell}}(0, r)$ between partial derivatives of the incident background and the Green function. The simplest choice of background is $h^{\text {bg }}=\alpha r^{\ell} \cos \ell \varphi$. While a Cartesian index contraction is not hard to do when the polarization is of order 1 or 2 , a general expression is difficult to come by in this way for high orders. Luckily, if one carries out the contraction in complex coordinates $(z, \bar{z})$ instead of $(x, y)$, a general expression is found easily. The result is that the effective object will respond to a background of the form $h^{\text {bg }}=\alpha r^{\ell} \cos \ell \varphi$ by creating the deformation

$$
\delta h(\boldsymbol{r})=-C^{(\ell)} \frac{2^{\ell} \ell !(\ell-1) ! \alpha}{4 \pi \sigma} \frac{\cos \ell \varphi}{r^{\ell}},
$$

which is derived in Appendix A. 
In the full theory, one has to solve the boundary value problem $\nabla^{2} \delta h=0$ for $r>R$ with the boundary condition $\left.h\right|_{r=R}=\left.\left(h^{\text {bg }}+\delta h\right)\right|_{r=R}=0 .{ }^{2}$ The full set of harmonic functions with square-integrable gradient has the form $r^{-\ell} \cos \ell \varphi$ with $\ell \in\{1,2, \ldots\}$. Hence the solution in the case of a background $h^{\text {bg }}=\alpha r^{\ell} \cos \ell \varphi$ is found to be

$$
\delta h(\boldsymbol{r})=-\alpha R^{2 \ell} \frac{\cos \ell \varphi}{r^{\ell}} .
$$

Comparison of Eqs. (12) and (13) yields the complete set of polarizabilities

$$
C^{(\ell)}=\frac{4 \pi R^{2 \ell} \sigma}{2^{\ell} \ell !(\ell-1) !}, \quad \ell=1,2,3 \ldots,
$$

for one object. For the sake of clarity, let us rewrite the complete $\Delta \mathcal{H}$ by restoring the particle labels $a$ :

$$
\Delta \mathcal{H}[h]=\frac{1}{2} \sum_{a} \sum_{\ell=1}^{\infty} C_{a}^{(\ell)}\left[\partial^{\ell} h\left(\boldsymbol{r}_{a}\right)\right]^{2},
$$

where $\partial^{\ell}$ denotes $\partial_{i_{1}} \partial_{i_{2}} \ldots \partial_{i_{\ell}}$. We now know the point-particle EFT Hamiltonian which-to quadratic order in the field-is rigorously equivalent to the full theory.

As stated earlier, we do not intend to address higher-thanquadratic terms in the effective theory here. However, we would like to stress that the matching procedure is essentially the same even if such terms are considered. The steps taken since the beginning of Sec. III do not rely on the responses being linear. What has been presented boils down to computing the one-point function $\langle\delta h(\boldsymbol{r})\rangle$ of the response $\delta h(\boldsymbol{r})$ of one isolated object to a background in the effective (point particle) and full theories. Generally, one matches $\operatorname{such} n$-point functions, or other observables that are functions or functionals of them, across both theories to fix the Wilson coefficients. For the linear case, it turned out to be very straightforward to fix the coefficients independently of each other by matching the aforementioned one-point function. If nonlinearities are also accounted for, one may need to match more $n$-point functions to obtain a sufficient number of linearly independent matching conditions to fix the values of the coefficients, and there may not be a simple choice of background that automatically decouples them. However, despite the tedium introduced by the nonlinearities, the procedure is not intrinsically problematic.

\section{B. Fluctuation-induced interactions}

With the effective theory established, we can now turn our attention to evaluating the cumulant expansion (6) explicitly. Taking powers of Eq. (15) one observes that the $q$ th cumulant in Eq. (6) involves $2 q$-point connected correlation functions. This can be represented as connected Feynman diagrams with $q$ vertices and two links coming out of each vertex (see Fig. 2, for instance), as one can see by Wick contraction of the $2 q$ point

\footnotetext{
${ }^{2}$ Strictly speaking, the boundary condition is $\left.h\right|_{r=R}=a+\boldsymbol{b} \cdot \boldsymbol{r}$ where $a$ and $\boldsymbol{b}$ are free parameters describing the height and tilt (if applicable) fluctuations of the colloid. Their function is to satisfy the boundary condition readily without the need for an induced response for those backgrounds that the colloid can align with.
}

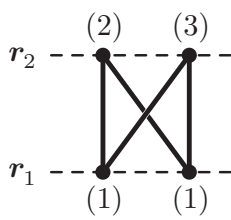

(a)

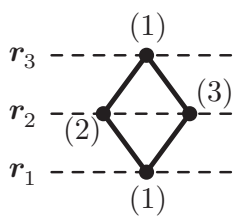

(b)

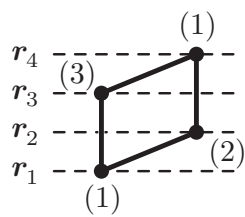

(c)
FIG. 2. Diagrams depicting pair (a), triplet (b), and quadruplet (c) interactions stemming from the fourth cumulant. Dashed lines represent the world lines of particles and have been labeled by the particle. The number in parentheses at each vertex shows the multipole order at the associated point in the scattering process. Each propagator is subject to $\ell_{1}+\ell_{2}$ partial derivatives, where $\ell_{1}$ and $\ell_{2}$ are the multipole orders at the vertices it connects. Consequently all these diagrams are interactions of order $2(1+2+3+1)=14$ in the inverse interparticle separations. Also note that we show only one of three possible four-body connections in (c), because our aim at this stage is just to work an example and not to be complete.

correlator. Every vertex contributes a factor of $\beta C^{(\ell)} / 2$ while every link affords a two-point correlator (or propagator) as in Eq. (7), acted upon by derivatives stemming from Eq. (15). Recalling that $C^{(\ell)} \sim \sigma$, one can see that there will be no factors of $\beta$ or $\sigma$ left in any cumulant; i.e., the dimensionless free energy $\beta F$ is purely a function of the spatial configuration of particles. Each correlator $\left\langle\partial^{\ell} h\left(\boldsymbol{r}_{a}\right) \partial^{m} h\left(\boldsymbol{r}_{b}\right)\right\rangle \sim \partial^{\ell} \partial^{m} G\left(\boldsymbol{r}_{a}, \boldsymbol{r}_{b}\right)$ brings a factor $\left|\boldsymbol{r}_{a}-\boldsymbol{r}_{b}\right|^{-(\ell+m)}$. Consequently, an interaction among $q$ multipoles of orders $\ell_{1}$ to $\ell_{q}$ will scale with a total of $-2\left(\ell_{1}+\ell_{2}+\cdots+\ell_{q}\right)$ powers of the separations between them. Accordingly, one knows how many multipole orders and how many cumulants must be retained, in order to achieve any given level of accuracy of an asymptotic expansion of the free energy, expressed in inverse powers of interparticle distances.

It is instructive to consider one example term from the cumulant expansion, to make these ideas clearer. The fourth cumulant will serve well since it contains pair, three-body, and four-body interactions, while the expressions are still not very cluttered. Let us first write the fourth cumulant directly from Eqs. (6) and (15):

$$
\begin{aligned}
\beta F^{(4)}:= & -\frac{\beta^{4}}{4 !} \sum_{\substack{a, b \\
c, d}} \sum_{\ell_{a}, \ell_{b}} \frac{C_{a}^{\left(\ell_{a}\right)} C_{b}^{\left(\ell_{b}\right)} C_{c}^{\left(\ell_{c}\right)} C_{d}^{\left(\ell_{d}\right)}}{2^{4}}\left\langle\left[\partial^{\ell_{a}} h\left(\boldsymbol{r}_{a}\right)\right]^{2}\right. \\
& \left.\times\left[\partial^{\ell_{b}} h\left(\boldsymbol{r}_{b}\right)\right]^{2}\left[\partial^{\ell_{c}} h\left(\boldsymbol{r}_{c}\right)\right]^{2}\left[\partial^{\ell_{d}} h\left(\boldsymbol{r}_{d}\right)\right]^{2}\right\rangle_{c} .
\end{aligned}
$$

The summations are over the particles and the multipoles on each. In this section we will discuss only a few terms embodied in these sums, chosen as instructive examples (shown in Fig. 2).

We will begin by considering the pair interaction between particles 1 and 2, depicted in Fig. 2(a). There are two factors one needs to compute: (1) the number of times the relevant product of polarizabilities, namely, $C_{1}^{(1)} C_{1}^{(1)} C_{2}^{(2)} C_{2}^{(3)}$, occurs in the sum (16), and (2) the multiplicity of the Wick contraction of the eight-point connected correlation function. The first of these factors is simply the multinomial coefficient $\left(\begin{array}{c}4 \\ 2,1,1\end{array}\right)=$ $4 ! / 2 ! 1 ! 1$ !, since what we are looking for is the factor in front of $a^{2} b c$ in the expansion of $(a+b+c+\cdots)^{4}$. Next, the multiplicity of the Wick contraction, or the diagram Fig. 2(a), 
is $2^{4}$ due to the possibility of flipping the legs of each vertex. ${ }^{3}$ Thus the pair interaction of Fig. 2(a) can be written as

$$
\begin{aligned}
\beta F[\text { Fig. 2(a)] }= & -\frac{1}{4 !}\left(\begin{array}{c}
4 \\
2,1,1
\end{array}\right) \frac{C_{1}^{(1)^{2}} C_{2}^{(2)} C_{2}^{(3)}}{(2 \sigma)^{4}} \\
& \times 2^{4} G_{i j k}^{12} G_{j k l}^{21} G_{l m n p}^{12} G_{m n p i}^{21},
\end{aligned}
$$

where the shorthand $G^{a b}=G\left(\boldsymbol{r}_{a}, \boldsymbol{r}_{b}\right)$ has been introduced. For completeness, we also present the result after doing the index contraction (see Appendix A) and substituting polarizabilities from Eq. (14):

$$
\beta F[\text { Fig. 2(a) }]=-6 \frac{R_{1}^{4} R_{2}^{10}}{r_{12}^{14}},
$$

where $r_{a b}=\left|\boldsymbol{r}_{a}-\boldsymbol{r}_{b}\right|$.

The triplet interaction depicted in Fig. 2(b) requires consideration of terms in Eq. (16), which involve the polarizabilities $C_{1}^{(1)}, C_{2}^{(2)}, C_{2}^{(3)}$, and $C_{3}^{(1)}$. This combination occurs $\left(\begin{array}{c}4 \\ 1,1,1,1\end{array}\right)$ times in Eq. (16), which means, also recalling that the multiplicity of the connection is $2^{4}$, that we obtain for the triplet interaction,

$$
\begin{aligned}
\beta F[\text { Fig. 2(b) }]= & -\frac{1}{4 !}\left(\begin{array}{c}
4 \\
1,1,1,1
\end{array}\right) \frac{C_{1}^{(1)} C_{2}^{(2)} C_{2}^{(3)} C_{3}^{(1)}}{(2 \sigma)^{4}} \\
& \times 2^{4} G_{i j k}^{12} G_{j k l}^{23} G_{\text {lmnp }}^{32} G_{m n p i}^{21} .
\end{aligned}
$$

After index contraction and substituting the polarizabilities, we have

$$
\beta F[\text { Fig. 2(b) }]=+12 \cos \varphi_{13}^{2} \frac{R_{1}^{2} R_{2}^{10} R_{3}^{2}}{r_{12}^{7} r_{23}^{7}},
$$

where $\varphi_{13}^{2}$ is the angle between $\boldsymbol{r}_{1}-\boldsymbol{r}_{2}$ and $\boldsymbol{r}_{2}-\boldsymbol{r}_{3}$, or in this case, the exterior angle of the triangle formed by the particles at $\boldsymbol{r}_{2}$. The quadruplet interaction of Fig. 2(c) is similarly found as

$$
\begin{aligned}
\beta F[\text { Fig. 2(c) }]= & -\frac{1}{4 !}\left(\begin{array}{c}
4 \\
1,1,1,1
\end{array}\right) \frac{C_{1}^{(1)} C_{2}^{(2)} C_{3}^{(3)} C_{4}^{(1)}}{(2 \sigma)^{4}} \\
& \times 2^{4} G_{i j k}^{12} G_{j k l}^{24} G_{l m n p}^{43} G_{m n p i}^{31} \\
= & -12 \cos \left(3 \varphi_{41}^{2}+4 \varphi_{14}^{3}\right) \frac{R_{1}^{2} R_{2}^{4} R_{3}^{6} R_{4}^{2}}{r_{12}^{3} r_{24}^{3} r_{43}^{4} r_{31}^{4}},
\end{aligned}
$$

with an analogous definition of the angles.

In this section we have tried to illustrate the Feynman rules of our expansion by specific examples. It should be clear that computing interactions is a straightforward exercise in

\footnotetext{
${ }^{3}$ It is generally $2^{q}$ with $q$ being the cumulant order, except for $q=2$, where it is 2 .
}

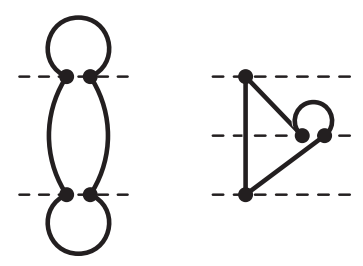

FIG. 3. Example diagrams with unphysical divergent self-energies. counting powers and diagrams relevant for the desired order in the inverse separation. The Green function products are easy to evaluate using complex coordinates as discussed in Appendix A, and they turn out to be proportional to a product of powers of the distances and the cosine of a combination of angles associated with the geometry of the particle configuration, determined by the multipole orders involved.

The reader may have realized that there seem to be divergent pair and triplet interactions in Eq. (16) due to one or more self-links in the form $\partial^{n} G(0)$ (see Fig. 3). These divergences are all power-like $\left(\sim r^{-n}\right.$ as $r \rightarrow 0$ where $\left.n \geqslant 2\right)$ and carry no physical information, as there is no nontrivial renormalization group (RG) flow. The rigorous way to deal with them is to add counterterms to the Hamiltonian, aiming to cancel the unphysical divergences from the free-energy expansion. However, since one can find all these counterterms by renormalization techniques, and the sole purpose of them is to remove the divergences from the free-energy expansion, the upshot of this systematic RG treatment is that the divergences can simply be dismissed. We have included Appendix $C$ where the removal of one such divergence by a counterterm is explicitly illustrated.

\section{Pair interactions}

Although every multibody order is included in the cumulant expansion (6), a systematic enumeration of all relevant diagrams lies beyond the scope of this article. However, if only two particles are involved, the solution can be worked out without too much trouble, and one can write the complete asymptotic expansion of the free energy.

Let us first establish that only even-numbered cumulants are relevant for pair interactions. The reason is that there is no physical (i.e., finite) pair term in odd-numbered cumulants. The easiest way to see this is to consider a pair diagram, i.e., one with connections between two world lines such as Fig. 2(a), with an odd number of vertices distributed along them: There is no possible connection (Wick contraction) without at least one self-link. As we discussed above, such contributions to the free energy are unphysical artifacts that can be removed rigorously. Hence, pair interactions exclusively stem from even-numbered cumulants.

To write the full expansion of the pair free energy, one must be able to do two things at an arbitrary cumulant order $2 s$ : (1) enumerate all contributing pair diagrams with $2 s$ vertices and (2) evaluate the diagrams, which involves doing the product of $2 s$ propagators [such as in Eq. (17), (20), or (21)] with any collection of multipole orders at the vertices. Luckily, both of these can be done. The propagator product is calculated in Appendix A. As for the enumeration of relevant diagrams, it is most suitable to again illustrate it with an example: The sixth cumulant contains the diagrams depicted in Fig. 4. These are all the connected pair diagrams with six vertices, free of unphysical self-energies. As one can see by starting from any one of the vertices and "stitching" the vertices in all possible ways, there are $3 ! 2 ! / 2=6$ of these diagrams, which generalizes to $g_{s}:=s !(s-1) ! / 2$ for the $(2 s)$-th cumulant (except that $g_{1}=1$ ). With each given collection of multipole orders at the vertices, the contribution of each diagram to the cumulant, or the propagator product, will be different (not in terms of power of $r$ but its prefactor) since the partial derivatives will generally be distributed differently on the 


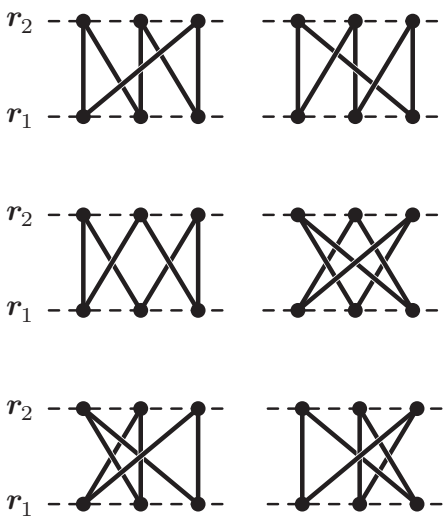

FIG. 4. Different "stitches" of the sixth cumulant pair interaction.

propagators. While this might seem to complicate things, remember that there is a sum over all possible collections of multipole orders out front [see Eq. (16)]. Thus, the effect of these $g_{s}$ different topologies is to repeat each scattering process $g_{s}$ times in the sum. Hence one can consider only one of the $g_{s}$ possible diagrams and multiply the result by $g_{s}$.

Taking everything together, the entire pair interaction can be written as

$$
\begin{aligned}
\beta F= & -\sum_{\left\{\ell_{i}\right\}}\left[\frac{1}{2 !}\left(\begin{array}{l}
2 \\
1
\end{array}\right) g_{1} \bigcap^{-}+\frac{1}{4 !}\left(\begin{array}{l}
4 \\
2
\end{array}\right) g_{2}\right. \\
& +\frac{1}{6 !}\left(\begin{array}{l}
6 \\
3
\end{array}\right) g_{3}
\end{aligned}
$$

where $\sum_{\left\{\ell_{i}\right\}}$ denotes a sum over all possible collections of multipoles at the vertices. Using the exact expressions for the polarizabilities, Eq. (14), and propagator products, Eq. (A11), to evaluate the diagrams, the pair interaction can be expressed in the following asymptotic expansion:

$$
\beta F=-\sum_{s=1}^{\infty} \frac{1}{s} \sum_{\ell_{1}, \ldots \ell_{2 s}} \prod_{i=1}^{2 s} \frac{\left(\ell_{i}+\ell_{i+1}-1\right) !}{\ell_{i+1} !\left(\ell_{i}-1\right) !} \frac{R_{1}^{2 L_{\mathrm{o}}} R_{2}^{2 L_{\mathrm{e}}}}{r^{2\left(L_{\mathrm{o}}+L_{\mathrm{e}}\right)}},
$$

where $\ell_{2 s+1}=\ell_{1}, L_{\mathrm{o}}=\sum_{\text {odd } i} \ell_{i}$ and $L_{\mathrm{e}}=\sum_{\text {even } i} \ell_{i}$. Assuming identical particle radii to reduce clutter, the first few terms of this expansion are

$$
\begin{aligned}
\beta F= & -\left(\frac{R}{r}\right)^{4}-4\left(\frac{R}{r}\right)^{6}-\frac{31}{2}\left(\frac{R}{r}\right)^{8} \\
& -60\left(\frac{R}{r}\right)^{10}-\frac{697}{3}\left(\frac{R}{r}\right)^{12}+\cdots .
\end{aligned}
$$

Note that obtaining these numbers does not involve anything more complicated than elementary algebra. The interaction up to order $r^{-70}$ has been plotted in Fig. 5. A cursory observation of the plot reveals that for (center-to-center) distances larger than $r \approx 4$, the lowest-order term in Eq. (24) suffices. However, for closer separations, the number of higher-order terms one should include increases rapidly. We demonstrate this in the inset to Fig. 5 where $r_{5 \%}(P)$ is defined as the separation where the series (24) terminated at $O\left(r^{-P}\right)$ achieves an accuracy of $5 \%$. As the dimensionless distance $d=r / R-2$ between the edges of the particles is decreased, the power

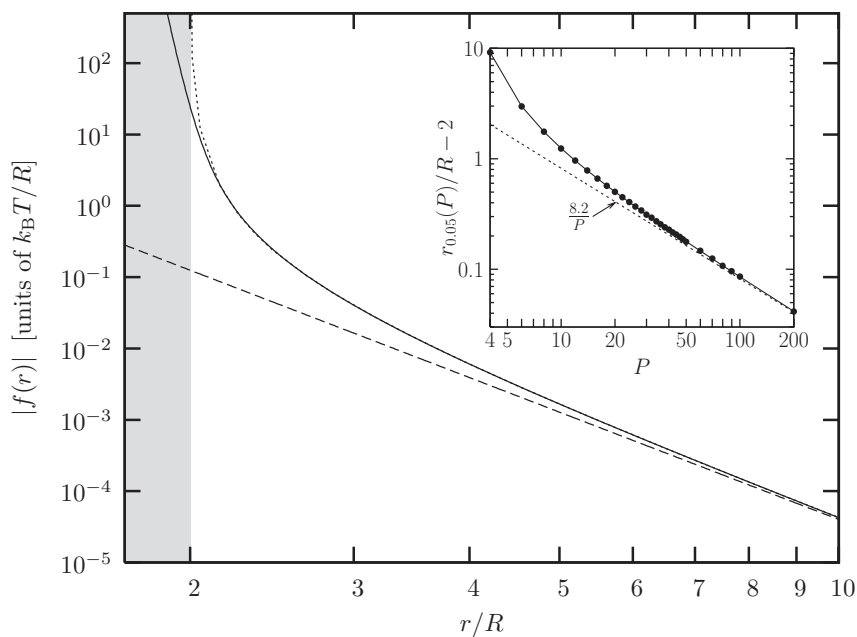

FIG. 5. Comparison to numerical results of Ref. [8] on the attractive force $f(r)=-\partial F / \partial r$. The dotted and solid curves correspond, respectively, to Ref. [8] and our results (truncated at order $r^{-70}$ in the free energy). The dashed line represents the lowest-order asymptotic solution, i.e., the dipole-dipole interaction. The inset depicts how the power $P$ of the highest-order term included in the expansion and the distance at which a $5 \%$ accuracy is achieved depend on each other.

where the series can be truncated and still achieve less than 5\% error appears to asymptotically obey $P \approx 8.2 d^{-1}$. From this we see, for instance, that if the surface-to-surface separation is a tenth of the particle radius, i.e., $d=0.1$, then one must retain all terms up to $O\left(r^{-82}\right)$ in order to achieve 5\% accuracy.

The first two terms of Eq. (24) are the dipole-dipole and dipole-quadrupole interactions also computed earlier by Lehle and Oettel [8]. The same publication also contains a quadrupole-quadrupole interaction of order $r^{-8}$, for the case of tiltable colloids, of magnitude -9 . Since the expansion (24) was developed under the assumption of frozen tilt degree of freedom, the magnitude of the $r^{-8}$ term includes additional interactions and is therefore different. Namely, these additional interactions are the quadrupole-octupole and dipole-dipoledipole-dipole interactions (Fig. 6) with respective magnitudes -6 and $-1 / 2$, such that the resulting total magnitude of the $r^{-8}$ term is $-9-6-1 / 2=-31 / 2$. Had we instead assumed the colloids to be free to tilt, their dipole polarizability $C^{(1)}$ would vanish-hence so would diagrams Fig. 6(b) and 6(c) - and the expansion (24) would have started with the quadrupole-quadrupole term $-9(R / r)^{8}$.

Lehle and Oettel also compute a numerical solution to the interaction for intermediate and close distances, where

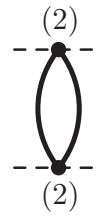

(a)

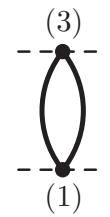

(b)

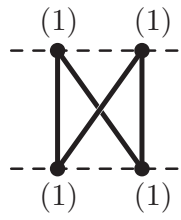

(c)
FIG. 6. (a) The quadrupole-quadrupole interaction $-9(R / r)^{-8}$. (b) The dipole-octupole interaction $-6(R / r)^{-8}$. (c) The dipoledipole-dipole-dipole interaction $-(1 / 2)(R / r)^{-8}$. 
the first few terms of the asymptotic expansion do not suffice [8]. As a confirmation of our result, we show in Fig. 5 the interaction found in Ref. [8] by performing the functional integrals numerically, with our expansion (23) up to order $r^{-70}$. The agreement is excellent, except upon approaching the contact distance $r=2 R$, where our truncated series remains finite while the numerical solution appears to grow without limit. The exact solution indeed diverges as

$$
-\beta F=\frac{\pi^{2}}{24 \sqrt{d}}+\frac{1}{4} \log \left(\frac{4 d}{\pi^{2}}\right)-\frac{96-\pi^{2}}{576} \sqrt{d}+O(d),
$$

where $d=(r-2 R) / R$ is a scaled surface-to-surface distance. The dominant term has previously been predicted by combining the exact result for two parallel lines with the Derjaguin approximation [8,32]; the subleading contributions cannot be extracted in this way but follow by expanding the exact analytical solution that one of us recently found by using conformal field theory techniques [33].

Since our formalism by construction produces an asymptotic expansion in the inverse separation, it is clearly not the preferred method when it comes to near-contact separations (see also inset in Fig. 5). However, it turns out that even our large distance expansion (23) is in a sufficiently manageable form to permit extraction of the leading order of (25) - including the prefactor-by some further analytical manipulation. We illustrate this in Appendix B. This, of course, constitutes an application of our expansion outside its region of highest analytical convenience, but it vividly illustrates that (1) our expansion can indeed be driven up to arbitrarily high order and that (2) the resulting predictions are correct up to the point where the exact solution diverges.

\section{Beyond the pair interaction}

When there are more than two particles involved, the free energy of interaction generally does not decompose into a sum of pair interactions but contains multibody interactions like the ones discussed in Sec. III B. As illustrated earlier, simple power counting reveals to what order in the separations a certain multibody interaction might contribute, and evaluating the interaction poses little difficulty.

Let us now discuss the leading-order triplet interaction. A dipole-dipole-dipole term from the third cumulant is the lowest-order triplet interaction conceivable. It is easy to see by power counting that this interaction would scale as $r_{12}^{-2} r_{23}^{-2} r_{31}^{-2}$. However, the strength of this interaction turns out to be identically zero. This is due to the harmonicity of the Green function of the problem and applies to any diagram in an oddnumbered cumulant (see Appendix A). Therefore, with this choice of surface Hamiltonian, many interactions that would seem to exist based on scaling arguments actually disappear. ${ }^{4}$

Since no triplet interaction exists in the third cumulant, one must look for the leading triplet term in the next. The lowest-order interaction is between induced dipoles and stems from the diagrams depicted in Fig. 7(a). We have already computed a subleading correction to this as an example in an earlier section, which involved higher-order multipoles. The leading order is similarly computed and found to be

$$
\begin{aligned}
\beta F_{\text {lead }}^{\text {trip }}= & -\frac{1}{4 !}\left(\begin{array}{c}
4 \\
1,2,1
\end{array}\right) \frac{C_{1}^{(1)} C_{2}^{(1)^{2}} C_{3}^{(1)}}{(2 \sigma)^{4}} 2^{4} G_{i j}^{12} G_{j k}^{23} G_{k l}^{32} G_{l i}^{21} \\
& + \text { cyclic permutations of }(123),
\end{aligned}
$$

which becomes, after evaluating the propagator product-by either direct computation or using Eq. (A11) — and substituting the polarizabilities from Eq. (14),

$$
\beta F_{\text {lead }}^{\text {trip }}=-\frac{R_{1}^{2} R_{2}^{4} R_{3}^{2}}{r_{12}^{4} r_{23}^{4}}-\frac{R_{1}^{2} R_{2}^{2} R_{3}^{4}}{r_{23}^{4} r_{31}^{4}}-\frac{R_{1}^{4} R_{2}^{2} R_{3}^{2}}{r_{31}^{4} r_{12}^{4}} .
$$

The fourth cumulant is also where the leading-order quadruplet interaction resides. This results from the diagrams shown in Fig. 7(b) among four induced dipoles. It follows as

$$
\begin{aligned}
\beta F_{\text {lead }}^{\text {quad }} & =-\frac{1}{4 !}\left(\begin{array}{c}
4 \\
1,1,1,1
\end{array}\right) \frac{C_{1}^{(1)} C_{2}^{(1)} C_{3}^{(1)} C_{4}^{(1)}}{(2 \sigma)^{4}} 2^{4}\left(G_{i j}^{12} G_{j k}^{23} G_{k l}^{34} G_{l i}^{41}+G_{i j}^{12} G_{j k}^{24} G_{k l}^{43} G_{l i}^{31}+G_{i j}^{13} G_{j k}^{32} G_{k l}^{24} G_{l i}^{41}\right) \\
& =2 R_{1}^{2} R_{2}^{2} R_{3}^{2} R_{4}^{2}\left[\frac{\cos \left(\varphi_{13}^{2}-\varphi_{24}^{3}+\varphi_{31}^{4}-\varphi_{42}^{1}\right)}{r_{12}^{2} r_{23}^{2} r_{34}^{2} r_{41}^{2}}+\frac{\cos \left(\varphi_{14}^{2}-\varphi_{23}^{4}+\varphi_{41}^{3}-\varphi_{32}^{1}\right)}{r_{24}^{2} r_{43}^{2} r_{31}^{2} r_{12}^{2}}+\frac{\cos \left(\varphi_{12}^{3}-\varphi_{34}^{2}+\varphi_{21}^{4}-\varphi_{43}^{1}\right)}{r_{32}^{2} r_{24}^{2} r_{41}^{2} r_{13}^{2}}\right]
\end{aligned}
$$

Depending on the desired level of accuracy, corrections to these interactions as well as further multiplets may be required. In this case, one simply identifies the relevant diagrams or interactions by power counting, and the computation that follows is fairly straightforward as shown.

\section{On anisotropic and flexible objects}

We have carried out our discussion under the assumption of rigid and circular particles. An obvious extension is toward

\footnotetext{
${ }^{4}$ This is not the case for a membrane, where the surface energy stems from bending elasticity [35].
}

particles with internal flexibility or nonaxisymmetric shapes. Although a thorough treatment of these extensions can be quite involved, there are nevertheless certain simple but rigorous remarks we can make at this stage.

Not much changes when the particles considered are flexible (or the constraints on their motion are not completely rigid). Since the boundary conditions at the circumference of the particles change accordingly, fixing the polarizabilities will require the solution of a different boundary value problem on the full theory side of the matching procedure, but the form of the effective Hamiltonian is exactly the same. The subsequent change in the values of the polarizabilities will result in different interaction strengths. However, this does not affect one's ability to identify features of the interaction, such 

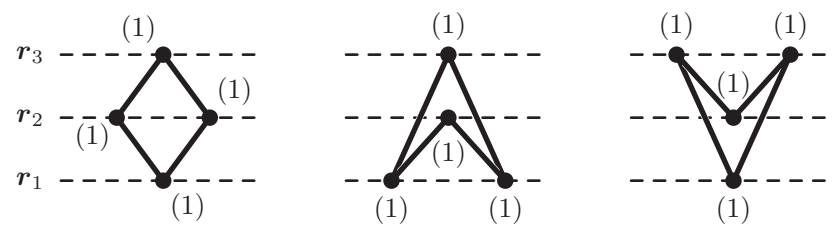

(a)

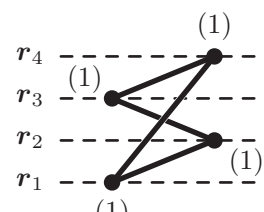

(1)
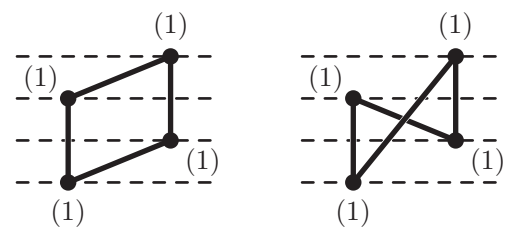

(b)

FIG. 7. (a) The fourth cumulant diagrams contributing to the leading triplet interaction. (b) The fourth cumulant diagrams contributing to the leading quadruplet interaction.

as what the leading power of inverse separations in a given multibody interaction is.

If the elastic properties of the particles are ever so slightly different from the surface, then the free-energy expansion acquires another smallness parameter intrinsic to the polarizabilities. This renders high cumulants in $\beta F$ negligible compared to the second, a so-called weak coupling [17] approximation. In such a case, being able to obtain a closedform result is more likely since only the second cumulant has to be taken into account. This is one of the limits considered in Ref. [19]. These authors restrict to two scatterings, analogously to truncating the cumulant expansion after the second, which is justified by the weakness of the perturbations.

In the case of an anisotropic particle, the response of the particle to incident backgrounds depends on its orientation, which is clearly not the case for the response given in Eq. (11). One now needs to consider polarizability tensors to describe the anisotropic response of a noncircular particle. The first few terms in the derivative expansion of a particle read

$$
\Delta \mathcal{H}_{a}=\left.\frac{1}{2}\left(h_{i} C_{i j}^{(1)} h_{j}+h_{i j} C_{i j k l}^{(2)} h_{k l}+\cdots\right)\right|_{r=r_{a}} .
$$

Fixing the polarizability tensors will require solving more complicated boundary value problems, but the lowest-order interaction will still stem from a pair of induced dipoles (tensor $C_{i j}^{(1)}$ ) and therefore scale as $r^{-4}$, no matter what the shape of the particle might be. Or if the particle is free to tilt, then the polarizability of any term involving an $h_{i}$ will vanish, thereby making the leading asymptotic interaction a quadrupole-quadrupole at order $r^{-8}$. These are indeed in line with the findings of Ref. [9], where explicit calculations of the leading order interactions between rigid ellipsoidal particles were presented.

\section{ON CORN FLAKES}

So far, we have focused on interactions initiated by thermal fluctuations and have thus restricted our study to particles that do not deform the film in its ground state. However, it is straightforward to include such effects in our formalism. This kind of situation naturally arises from an irregular three-phase contact line between a colloid and the fluid-fluid interface it is trapped at [34]. Macroscopically, we see corn flakes perturbing the surface of a bowl of milk in a similar way. Again, one would expect (and can indeed observe) that once these deformations overlap, they give rise to aggregation of the flakes.

These perturbations are treated as "permanent charges" in the effective theory, beside the induced charges arising from constraints. The effective theory incurs local linear terms in addition to the quadratic ones upon this modification. The easiest way to determine these linear terms is again through an argument involving the response of each particle to a background. Consider a particle whose contact line height profile has a nontrivial multipole $h^{\mathrm{cl}}(\varphi)=\eta_{\ell} \cos \left(\ell \varphi-\ell \alpha_{\ell}\right)$ around its center with a phase angle $\alpha_{\ell}$. When a background $h^{\mathrm{bg}}(\boldsymbol{r})=\eta_{\ell} R^{-\ell} r^{\ell} \cos \left(\ell \varphi-\ell \alpha_{\ell}\right)$ is incident on this particle, the boundary condition $\left.\left(h^{\text {in }}=h^{\text {out }}\right)\right|_{r=R}$ is automatically satisfied by the fact that $h^{\mathrm{bg}}(R, \varphi)=h^{\mathrm{cl}}(\varphi)$, and hence no net response will be triggered. Another way to phrase this is that the field of the permanent charge and the induced response to this specific background eliminate each other. Therefore, if this "preferred background" is denoted by $p^{(\ell)}(\boldsymbol{r})$, the local linear terms representing the permanent charges can be encoded into the quadratic induced charge terms as a shift:

$$
\Delta \mathcal{H}[h]=\frac{1}{2} \sum_{a, \ell} C_{a}^{(\ell)}\left[\partial^{\ell} h\left(\boldsymbol{r}_{a}\right)-\partial^{\ell} p^{(\ell)}\left(\boldsymbol{r}_{a}\right)\right]^{2} .
$$

Note that the values of the polarizability coefficients are the same, whether the preferred shape of the particles are flat or curved, as long as the constraints are the same. Notice that in the absence of (vertical) external forces, the vertical movement of a particle is free and hence $C^{(0)}=0$. Similarly, in the absence of (horizontal) external torques, the tilt motion is free and $C^{(1)}=0$. In the following, we will assume that this is the case, and therefore the multipole expansion starts at $\ell=2$.

From Eq. (30) one sees that the linear terms in the effective Hamiltonian are

$$
-\sum_{a, \ell} C_{a}^{(\ell)} \partial^{\ell} p^{(\ell)}\left(\boldsymbol{r}_{a}\right) \partial^{\ell} h\left(\boldsymbol{r}_{a}\right),
$$

which encode the permanent sources

$$
-\sum_{a, \ell}(-)^{\ell} C_{a}^{(\ell)} \partial^{\ell}\left[\delta\left(\boldsymbol{r}-\boldsymbol{r}_{a}\right) \partial^{\ell} p^{(\ell)}\left(\boldsymbol{r}_{a}\right)\right] .
$$

In the diagrammatic expansion, the linear terms correspond to vertices with only one link attached. Therefore, interactions involving permanent charges are open diagrams initiating and terminating at these. Such diagrams have one link less but the same number of vertices compared to the fluctuation-induced interactions, and power counting shows the free energy due to them will scale as $F \sim \sigma R^{2}$, i.e., with no temperature dependence. These ground-state contributions together with the fluctuation part, $\sim k_{\mathrm{B}} T$, of the previous section result in a free energy of the form $F=a_{0}+a_{1} T$, which allows us to view this separation into ground-state energy and fluctuation correction as equivalent to a separation into the energy and entropy terms in $F=E-T S$. Observe that this equivalence would not hold had we considered anharmonicities in the 


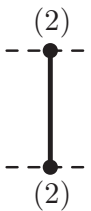

(a)

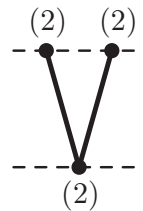

(b)

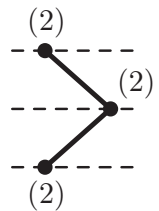

(c)
FIG. 8. (a) The direct interaction $E^{(4)} \sim r^{-4}$ between two permanent quadrupoles. (b) The pair interaction $E^{(8)} \sim r^{-8}$ between a permanent quadrupole and the quadrupole it induces on the other particle. This is the lowest-order energetic pair interaction that involves scattering from an induced charge. (c) At the same order as diagram (b), there also exists a triplet interaction $E_{\text {trip }}^{(8)}$ involving two separate permanent charges and one induced charge.

theory and thus furnished the free energy with contributions higher than linear order in the temperature.

For the sake of clarity, let us consider a pair of particles with a saddle-shaped contact line deformation. That is, each particle will have a preferred background of the form $p(\boldsymbol{r})=$ $(1 / 2) k r^{2} \cos (2 \varphi-2 \alpha)$ around its center, where the angle $\alpha$ describes the orientation of the saddle. For a colloid under no external vertical force (e.g., gravity) or horizontal torque, this quadrupole deformation is the lowest-order multipole contact line irregularity that can occur. The linear part of the effective theory follows as $-\sum_{a} C_{a}^{(2)} p_{i j}\left(\boldsymbol{r}_{a}\right) h_{i j}\left(\boldsymbol{r}_{a}\right)$, where

$$
p_{i j}\left(\boldsymbol{r}_{a}\right)=k_{a}\left[\begin{array}{rr}
\cos 2 \alpha_{a} & -\sin 2 \alpha_{a} \\
-\sin 2 \alpha_{a} & -\cos 2 \alpha_{a}
\end{array}\right] .
$$

Notice the simple geometric interpretation of $k_{a}$ as the magnitudes of each principal curvature of the saddles.

The leading interaction stems from the second cumulant as a direct interaction between the two permanent quadrupoles, Fig. 8(a), easily found as

$$
\begin{aligned}
E^{(4)} & =-\frac{1}{2 !}\left(\begin{array}{c}
2 \\
1,1
\end{array}\right) \frac{C_{1}^{(2)} C_{2}^{(2)}}{\sigma} p_{i j}\left(\boldsymbol{r}_{1}\right) G_{i j k l}^{12} p_{k l}\left(\boldsymbol{r}_{2}\right) \\
& =-3 \pi \sigma \cos \left(2 \alpha_{1}+2 \alpha_{2}\right) \frac{k_{1} R_{1}^{4} R_{2}^{4} k_{2}}{r^{4}},
\end{aligned}
$$

where the pair was assumed to lie on the $x$ axis to declutter the expression. The functional dependence of the interaction implies both an attractive force and vertical torques on the saddle-like particles. The same potential was found by Stamou et al. [34]. We have seen earlier that, between colloids that are free to tilt and fluctuate vertically, the leading fluctuationinduced interaction is of order $r^{-8}$, which can compete with the interaction (34) only at close separations.

Although we have restricted to a toy problem where the only permanent deformation is quadrupolar, it should be obvious how one would handle higher-order multipoles of the contact line irregularity for accuracy at closer separations. However, one should note that the true interaction potential is not merely a superposition of direct interactions between permanent charges, similar to Fig. 8(a), but includes scatterings from induced charges, such as Fig. 8(b). The former is equivalent to assuming the total shape of the surface is given by a superposition of the deformations caused by each particle independently of every other, i.e., neglecting their boundary conditions, which is why it misses induced charges. Such a superposition approximation is captured by the second cumulant and holds up to the order the next cumulant begins to contribute. This is the order $r^{-8}$, where the third cumulant contributes the diagram in Fig. 8(b), evaluating to the repulsion

$$
E^{(8)}=\frac{9}{2} \pi \sigma\left(k_{1}^{2} R_{1}^{4}+k_{2}^{2} R_{2}^{4}\right) \frac{R_{1}^{4} R_{2}^{4}}{r^{8}},
$$

which is the lowest-order pair interaction that involves a scattering from an induced charge. At the same order, one observes the first multibody interaction as well, which is depicted in Fig. 8(c), and evaluates to $E_{\text {trip }}^{(8)}=E_{123}^{(8)}+E_{231}^{(8)}+$ $E_{312}^{(8)}$, where

$$
E_{123}^{(8)}=9 \pi \sigma \frac{k_{1} R_{1}^{4} R_{2}^{4} R_{3}^{4} k_{3}}{r_{12}^{4} r_{32}^{4}} \cos \left(4 \varphi_{13}^{2}+2 \alpha_{1}-2 \alpha_{2}\right),
$$

and so on. If the particles possess higher multipole order contact line irregularities, merely taking these into account within a superposition approximation does not capture every possible correction systematically, since it misses contributions such as Fig. 8(b) and 8(c) by construction.

To summarize, although we primarily use the EFT formalism to facilitate the evaluation of a partition function, the ground-state interactions mediated by the surface can easily be calculated as well. One can readily encode the permanent deformations imposed by the colloids as linear terms in the effective theory, upon which the ground-state energy strictly separates from the fluctuation correction. Computations of this energy that rely upon the superposition of perturbations caused by the particles only hold for direct interactions of the second cumulant.

\section{CONCLUSION}

We have presented and discussed a formalism to compute the surface-mediated forces between rigid disks trapped at an interface, based on effective field theory. This formalism reduces each finite-sized particle to a point, which greatly simplifies computations. Still, these points are by explicit construction equipped with all the information necessary to capture their finite-sized counterpart's behavior, and hence the treatment is not approximate. In particular, their size is not simply recovered as the continuum theory's ad hoc ultraviolet cutoff-an approach that can neither conceptually satisfy nor be plausibly extended to more than one particle radii.

The extension from the free-surface theory to the effective theory is achieved by the addition of a derivative expansion $\Delta \mathcal{H}$, with extra terms localized at the centers of the particles, encoding the constraints they enforce on the fluctuations as induced capillary multipoles. Symmetries determine the form of this extension to the Hamiltonian, while one still has to fix the values of undetermined polarizability coefficients via a matching procedure. However, one gains insight into the interaction free energy even without fixing these coefficients, such as the leading-order distance dependence of the force or the vanishing of certain many-body interactions due to the 
kernel of the theory. In this article we focused on circularly symmetric particles, which allowed us to determine the effective Hamiltonian completely, i.e., to all derivative orders. However, in cases where this is not possible and a certain level of accuracy-in powers of $r$-is desired, knowing only a fraction of all the polarizability coefficients will suffice.

After the effective theory has been established, the interaction free energy is then computed in a cumulant expansion. $\mathrm{N}$ body interactions are identified as Feynman diagrams linking $N$ world lines and are computed in a fairly straightforward manner. The two expansions, the cumulant expansion and that in the local derivatives of the field at the inclusions, afford a transparent interpretation of every term that contributes to the interaction as a scattering process between various multipole moments of the induced charges, similar in spirit to Ref. [14]. Unless both of these expansions are performed, consistent results cannot be achieved. Also, owing to simplifications due to the harmonicity of the theory, the number of dimensions, and the symmetry of the particles, a general expression for any possible diagram was obtained in Appendix A. We have used this expression to develop a full asymptotic expansion of the pair interaction. It was possible to partially sum up the expansion. We believe a complete summation of the series to obtain a closed-form expression may be possible as well, though we have not succeeded here.

As we have discussed, an extension to nonrigid and noncircular objects entails no major change in the formalism. The former only affects the strength of the polarizability coefficients and therefore the interactions, while the latter requires consideration of tensor polarizabilities, and both fixing their values and computing interactions become a bit more involved.

Moreover, we have presented results on ground-state interactions between the particles as well as the fluctuation-induced interactions. This required us to add permanent charges to the effective theory, which is simply achieved by shifting the zero of the induced charge terms. The ground-state interactions then strictly separate from the fluctuation corrections and can be computed in the same manner we discussed for the fluctuationinduced forces. In addition to deriving the lowest-order pair interaction, in agreement with known results, we illustrated how corrections - higher order or multibody - are computed.

Note that the same formalism can be used to treat surfaces of different characters, i.e., different free-surface Hamiltonians. A relevant example is the problem of surface-mediated interactions between inclusions on a biological membrane, which we will treat in a future publication [35]. Additionally, fluctuation corrections of higher-than-linear order in $k_{\mathrm{B}} T$ can be systematically studied by increasing the anharmonicity of the theory, e.g., relaxing the weak deformation assumption on the interface or the constant footprint of the particles.

\section{APPENDIX A: INDEX CONTRACTIONS IN COMPLEX COORDINATES}

In this Appendix we will go into details of how to perform the index contractions encountered in computing induced fields and propagator products by transforming to complex coordinates, $z=x+\mathrm{i} y$ and $\bar{z}=x-\mathrm{i} y$. We first note that in Cartesian coordinates, covariant and contravariant elements of tensors are identical; i.e., the metric is the identity. Therefore we have not distinguished between them and have written all indices as subscripts in the main body of the article. However, now that we are considering a change of coordinates, we will use a covariant notation instead. For example, Eq. (11) will read

$$
\begin{aligned}
\delta h(\boldsymbol{r}) & =-\frac{C^{(\ell)}}{\sigma} h_{i_{1} \cdots i_{\ell}}^{\mathrm{bg}}(0) G^{i_{1} \ldots i_{\ell}}(0, \boldsymbol{r}) \\
& =-\frac{C^{(\ell)}}{\sigma} g^{i_{1} j_{1}} \cdots g^{i_{\ell} j_{\ell}} h_{i_{1} \cdots i_{\ell}}^{\mathrm{bg}}(0) G_{j_{1} \cdots j_{\ell}}(0, \boldsymbol{r}),
\end{aligned}
$$

where $g^{i j}$ is the (inverse) metric tensor.

From the embedding $x=(1 / 2)(z+\bar{z}), y=(1 / 2 \mathrm{i})(z-\bar{z})$ the tangent vectors $\vec{e}_{z}=(1 / 2)(1,-\mathrm{i})$ and $\vec{e}_{\bar{z}}=(1 / 2)(1, \mathrm{i})$ follow. Then the metric tensor $g_{i j}=\vec{e}_{i} \cdot \vec{e}_{j}$ and its inverse are found as

$$
g_{i j}=\frac{1}{2}\left(\begin{array}{ll}
0 & 1 \\
1 & 0
\end{array}\right), \quad g^{i j}=2\left(\begin{array}{ll}
0 & 1 \\
1 & 0
\end{array}\right) .
$$

Since the metric is constant, the Christoffel symbols vanish, and therefore covariant derivatives are just partial derivatives (and hence also commute).

The reason why it is useful to go to complex coordinates is as follows. The index contractions needed for our computations all involve partial derivatives of the harmonic Green function, which is

$$
G\left(z, z^{\prime}\right)=-\frac{1}{4 \pi} \log \left(z-z^{\prime}\right)\left(\bar{z}-\bar{z}^{\prime}\right)
$$

in complex coordinates. It is easily seen that

$$
\partial_{z} \partial_{\bar{z}} G=0,
$$

which is equivalent to the harmonic property of the Green function since $\nabla^{2} G=g^{i j} G_{i j}=4 \partial_{z} \partial_{\bar{z}} G$. This is a useful property because, when expressed in complex coordinates, all index combinations involving an alternation of indices on $G$ vanish from the contraction, leaving only terms that involve either

$$
\frac{\partial^{n} G}{\partial z^{n}}=\frac{(n-1) !}{4 \pi} \frac{1}{\left(z^{\prime}-z\right)^{n}}
$$

or its complex conjugate. This property and the simple form of the metric tensor (A2) simplify things greatly, allowing us to express products involving an arbitrary number of indices and factors.

The computation of the induced deformation, Eq. (11) or Eq. (A1), is now as follows: The background $h^{\text {bg }}=\alpha r^{\ell} \cos \ell \varphi$ can be rewritten in complex coordinates as $h^{\mathrm{bg}}=(\alpha / 2)$ $\left(z^{\ell}+\bar{z}^{\ell}\right)$. Using Eqs. (A2) and (A4) we find

$$
\begin{aligned}
\delta h(\boldsymbol{r}) & =-\frac{C^{(\ell)}}{\sigma} h_{i_{1} \cdots i_{\ell}}^{\mathrm{bg}}(0) G^{i_{1} \cdots i_{\ell}}(0, z) \\
& =-\frac{C^{(\ell)}}{\sigma} g^{i_{1} j_{1}} \cdots g^{i_{\ell} j_{\ell}} h_{i_{1} \cdots i_{\ell}}^{\mathrm{bg}}(0) G_{j_{1} \cdots j_{\ell}}(0, z) \\
& =-\frac{C^{(\ell)}}{\sigma} 2^{\ell} \partial_{\bar{z}}^{\ell} h^{\mathrm{bg}}(0) \partial_{z}^{\ell} G(0, z)+\text { c. c. }
\end{aligned}
$$


Note that the index contraction, which generally has $2^{\ell}$ terms, was reduced to only two terms, owing to the property $\partial_{z} \partial_{\bar{z}} G=$ 0. Now, using Eq. (A5) the result we used in Eq. (12) can be found:

$$
\begin{aligned}
\delta h(\boldsymbol{r}) & =-\frac{C^{(\ell)}}{\sigma} 2^{\ell} \frac{\alpha \ell !}{2} \frac{(\ell-1) !}{4 \pi}\left(\frac{1}{z^{\ell}}+\frac{1}{\bar{z}^{\ell}}\right) \\
& =-C^{(\ell)} \frac{2^{\ell} \ell !(\ell-1) ! \alpha}{4 \pi \sigma} \frac{\cos \ell \varphi}{r^{\ell}} .
\end{aligned}
$$

Note that one has to pay attention to which argument of the Green function is differentiated so as to avoid sign errors.
The other point where we encounter index contractions is in propagator products such as

$$
\partial^{\ell_{a}} \partial^{\ell_{b}} G^{a b} \partial^{\ell_{b}} \partial^{\ell_{c}} G^{b c} \partial^{\ell_{c}} \partial^{\ell_{d}} G^{c d} \partial^{\ell_{d}} \partial^{\ell_{a}} G^{d a},
$$

where $\partial^{\ell}$ is a shorthand for $\partial_{i_{1}} \partial_{i_{2}} \ldots \partial_{i_{\ell}}$. To make the underlying algebra more transparent, let us first look at this product of four propagators, before we generalize to an arbitrary number. Similarly to the derivation of Eqs. (A6) and (A7), and defining $z_{a b}=z_{a}-z_{b}$, this product can be written as

$$
\begin{aligned}
(\mathrm{A} 8)= & 2^{\ell_{a}+\ell_{b}+\ell_{c}+\ell_{d}} \partial_{z}^{\ell_{a}} \partial_{z}^{\ell_{b}} G\left(z_{a b}\right) \partial_{\bar{z}}^{\ell_{b}} \partial_{\bar{z}}^{\ell_{c}} G\left(z_{b c}\right) \\
& \times \partial_{z}^{\ell_{c}} \partial_{z}^{\ell_{d}} G\left(z_{c d}\right) \partial_{\bar{z}}^{\ell_{d}} \partial_{\bar{z}}^{\ell_{a}} G\left(z_{d a}\right)+\text { c.c. }
\end{aligned}
$$

Now we substitute from Eq. (A5) to find

$$
\begin{aligned}
(\mathrm{A} 8) & =\frac{\left(\ell_{a}+\ell_{b}-1\right) !}{4 \pi} \frac{\left(\ell_{b}+\ell_{c}-1\right) !}{4 \pi} \frac{\left(\ell_{c}+\ell_{d}-1\right) !}{4 \pi} \frac{\left(\ell_{d}+\ell_{a}-1\right) !}{4 \pi} \frac{(-2)^{\ell_{a}+\ell_{b}+\ell_{c}+\ell_{d}}}{z_{a b}^{\ell_{a}+\ell_{b}} \bar{z}_{b c}^{\ell_{c}+\ell_{c}} z_{c d}^{\ell_{c}+\ell_{d}} \bar{z}_{d a}^{\ell_{d}+\ell_{a}}}+\text { c.c. } \\
& =\frac{\left(\ell_{a}+\ell_{b}-1\right) !\left(\ell_{b}+\ell_{c}-1\right) !\left(\ell_{c}+\ell_{d}-1\right) !\left(\ell_{d}+\ell_{a}-1\right) !}{(4 \pi)^{4} 2^{-\left(\ell_{a}+\ell_{b}+\ell_{c}+\ell_{d}+1\right)}} \frac{\cos \left(\ell_{b} \varphi_{a c}^{b}-\ell_{c} \varphi_{b d}^{c}+\ell_{d} \varphi_{c a}^{d}-\ell_{a} \varphi_{d b}^{a}\right)}{r_{a b}^{\ell_{a}+\ell_{b}} r_{b c}^{\ell_{b}+\ell_{c}} r_{c d}^{\ell_{c}+\ell_{d}} r_{d a}^{\ell_{d}+\ell_{a}}},
\end{aligned}
$$

where $z_{a b}=: r_{a b} \mathrm{e}^{\mathrm{i} \varphi_{a b}}$ and $\varphi_{a c}^{b}:=\varphi_{a b}-\varphi_{b c}$ is the angle between $\boldsymbol{r}_{a}-\boldsymbol{r}_{b}$ and $\boldsymbol{r}_{b}-\boldsymbol{r}_{c}$, etc. It is obvious how this result generalizes to the product of an arbitrary number $q$ of propagators:

$$
2 \operatorname{Re} \prod_{i=1}^{q} \frac{2^{\ell_{i}}\left(\ell_{i}+\ell_{i+1}-1\right) !}{4 \pi} \frac{\mathrm{e}^{\mathrm{i}(-)^{i} \ell_{i} \varphi_{a_{i-1} a_{i+1}}}}{r_{a_{i} a_{i+1}}^{\ell_{i}+\ell_{i+1}}}
$$

with $\ell_{q+1}=\ell_{1}$. We use this formula to evaluate many interactions in Sec. III B, most noticeably in Eq. (23) where a pair interaction is considered and thus the angles are zero.

Last, we would like to revisit a statement we made in Sec. III B 2, namely, that the free-energy expansion (6) for the surface tension Hamiltonian has identically vanishing odd cumulants. This is easily seen to hold by observing that it is not possible to write a nonzero expression analogous to (A9) for an odd number of propagators, due to the property (A4) and the off-diagonal form of the metric. As it rests on Eq. (A4), or $\nabla^{2} G=0$, this property of all odd-numbered cumulants vanishing is special to the harmonic free-surface Hamiltonian.

\section{APPENDIX B: NEAR-CONTACT ASYMPTOTICS}

In problems where the field equation, the boundary conditions, etc., are more complicated than those considered in this article, one generally does not expect to calculate the cumulant expansion in its entirety. However, in this specific case, it is possible to obtain an exact and complete expansion: Eq. (23). While finding a closed form for the whole series or at least the expansion coefficients is a difficult task, considerable simplifications turn out to be possible. The inner $2 s$-fold sum of Eq. (23) has the form of a so-called binomial cycle [36], and it is possible to perform all but one of the sums adapting from Ref. [36]. Defining $u=R^{2} / r^{2}$ for the case of identical radii, Eq. (23) reduces to

$$
\beta F=-\sum_{s, n=0}^{\infty}\left(\begin{array}{c}
2 n \\
n
\end{array}\right) \frac{u^{2 s+n+2} \mathcal{F}\left(-n, 1 ;-2 n ; \frac{f_{2 s}(u)}{f_{2 s+1}(u)}\right)}{(s+1) f_{2 s+1}(u) f_{2 s}(u)},
$$

where $\mathcal{F}(a, b ; c ; x)$ is the hypergeometric function [37] and the polynomials $f_{q}(u)$ have the recursion relation

$$
f_{q}(u)=f_{q-1}(u)-u f_{q-2}(u),
$$

with $f_{0}=f_{-1}=1$, related to continued fractions. Also note that the summation index $s$ merely labels cumulants and is related to the cumulant order $q$ as $q=2(s+1)$. This already amounts to a big practical improvement over Eq. (23), because Eq. (B1) can be much more efficiently expanded in powers of inverse distance.

From the recursion relation of $f_{q}$, one can also derive the expression

$$
f_{q}(u)=\sum_{i=0}^{\left\lfloor\frac{q+1}{2}\right\rfloor}\left(\begin{array}{c}
q+1-i \\
i
\end{array}\right)(-u)^{i},
$$

where $\lfloor\cdot\rfloor$ denotes the integer part, or better still, recognize (in our case, MATHEMATICA did this for us) its closed form as

$$
f_{q}(u)=\frac{\chi^{q+2}-1}{\left(\chi^{2}-1\right)\left(\chi^{2}+1\right)^{q}},
$$

where $\chi(u)=(1-\sqrt{1-4 u}-2 u) / 2 u$. Thanks to the closedform expression for $f_{q}$, one can show that in the interval $0<u<1 / 4$ (i.e., from asymptotically separated to osculating disks, recalling $\left.u=R^{2} / r^{2}\right)$, the ratio $f_{2 s}(u) / f_{2 s+1}(u)<2$, 
which is the condition for the hypergeometric function in Eq. (B1) to converge as $n \rightarrow \infty$ :

$$
\lim _{n \rightarrow \infty} \mathcal{F}(-n, 1 ;-2 n ; x)=\left(1-\frac{x}{2}\right)^{-1} .
$$

One can then use Eq. (B5) to isolate the leading divergence in each cumulant $q=2(s+1)$ in Eq. (B1) as the $n \rightarrow \infty$ tail of the inner sum and obtain the resulting divergence in $\beta F$ as

$$
\beta F_{\infty} \sim-\sum_{s=0}^{\infty} \frac{u^{2 s+2} \sum_{n=0}^{\infty}\left(\begin{array}{c}
2 n \\
n
\end{array}\right) u^{n}}{(s+1) f_{2 s}(u)\left[f_{2 s+1}(u)-\frac{f_{2 s}(u)}{2}\right]} .
$$

The symbol " " reminds us that this captures only the leadingorder asymptotic divergence.

After noting that $\sum_{n=0}^{\infty}\left(\begin{array}{c}2 n \\ n\end{array}\right) u^{n}=(1-4 u)^{-\frac{1}{2}}$, we will define the (scaled) surface-to-surface separation $d$ between the disks as $u=1 /(d+2)^{2}$ and reexpress Eq. (B6) for $d \ll 1$ using Eq. (B4) in order to obtain the leading divergence in $\beta F_{\infty}$ :

$$
\beta F_{\infty} \sim-\frac{1}{4 \sqrt{d}} \sum_{s=1}^{\infty} \frac{1}{s^{2}}=-\frac{\zeta(2)}{4 \sqrt{d}}=-\frac{\pi^{2}}{24 \sqrt{d}},
$$

in agreement with Eq. (25).

The crudeness of isolating the divergent part only allows one to obtain the leading-order proximity asymptotics. In a recent preprint [33] one of us used conformal field theory to exactly compute the partition function of a bosonic field on a plane with two holes. This is isomorphic to the problem of two disks on a film studied in this article, and the interaction can in fact be written in closed form (it involves a Dedekind $\eta$ function). Its expansion at large distances reproduces our EFT series, while an expansion at contact leads to Eq. (25).

We again point out that computing the close distance divergence is not the most obvious application for our specific EFT implementation (which is built on a large distance expansion), but it nevertheless illustrates the power of the formalism quite vividly.

\section{APPENDIX C: CANCELLATION OF DIVERGENCES BY COUNTERTERMS}

In this Appendix we will illustrate how self-energy divergences can be removed by counterterms. While this is, to some extent, a standard textbook affair, we include it in order to illustrate the mechanism in the presently relevant context for readers not necessarily familiar with (quantum) field theory.

First, let us clarify which divergences are of interest here. Purely numerical divergences, such as those of the first cumulant, get absorbed in the definition of the free-surface energy (i.e., no particles); they do not refer to the relative positions of the particles and thus do not affect interaction energies. Similar trivial divergences occur in every cumulant order and do not require any further discussion. The more interesting divergences are those that multiply interactions between particles and thus cannot be lumped together with the free-surface energy.

The appearance of these divergences is due to the very construction of an effective field theory. The self-interactions necessarily involve the short-distance (high-energy) physics, through $G(0)$, where the theory is inadequate; especially in field theories of condensed matter, we know that the continuum description breaks down below a lattice spacing or an equivalent short length scale. But the effect of the short distance physics on large length scale (low-energy) observables, such as the polarizabilities $C^{(\ell)}$, is to renormalize (or "dress") their values. The values we obtained for these polarizabilities, through a procedure that has nothing to do with the short distance physics of the field, are therefore renormalized values. On the other hand, if a field theory is to make finite predictions on (low-energy) physical observables, the renormalized couplings must be accompanied by counterterms, or equivalently the coupling constants must be restored to their "bare" values. However, since the counterterms exist for the sole purpose of canceling these ultraviolet divergences in the values for physical observables, it is legitimate not to explicitly write the counterterms, but discard such divergences on these grounds when encountered, as we did in this article. We will nevertheless provide an example below.

For the sake of simplicity, let us assume two particles possessing only a dipole polarizability described by the Hamiltonian

$$
\Delta \mathcal{H}=\frac{1}{2} C_{1} h_{i}^{2}\left(\boldsymbol{r}_{1}\right)+\frac{1}{2} C_{2} h_{i}^{2}\left(\boldsymbol{r}_{2}\right) .
$$

It is not hard to see that the divergences in the first and second cumulants, $\langle\beta \Delta \mathcal{H}\rangle_{\mathrm{c}}$ and $-(1 / 2)\left\langle(\beta \Delta \mathcal{H})^{2}\right\rangle_{\mathrm{c}}$, are of the trivial sort; i.e., those that do not involve the distance between particles. However, in the third cumulant we encounter a divergent pair free energy

$$
\frac{1}{3 !}\left(\begin{array}{l}
3 \\
2
\end{array}\right) \frac{C_{1}^{2} C_{2}}{(2 \sigma)^{3}} 8 G_{i j}^{11} G_{j k}^{12} G_{k i}^{21},
$$

as well as a similar term with the labels 1 and 2 interchanged. Using the fact that $\partial_{i} \partial_{j} G(0)=(1 / 2) \delta_{i j} \nabla^{2} G(0)$, we can rewrite (C2) as

$$
\frac{1}{2}\left[\frac{C_{1}^{2}}{2 \sigma^{2}} \nabla^{2} G(0)\right] \frac{C_{2}}{\sigma} G_{j k}^{12} G_{k j}^{21},
$$

where it is apparent that this energy scales as $\delta(0) / r^{4}$. The way the factors were arranged in (C3) makes it easy to identify what counterterm should be added to the Hamiltonian to negate this unphysical divergence: One might check that the physical pair energy between these two particles at the second cumulant has exactly the form of (C3), except that the factor in square brackets is $C_{1} / \sigma$. This means that if one adds to $\Delta \mathcal{H}$ the counterterm

$$
\mathcal{H}_{\text {counter }}=\frac{1}{2} \frac{C_{1}^{2}}{2 \sigma} \nabla^{2} G(0) h_{i}^{2}\left(\boldsymbol{r}_{1}\right),
$$

it produces a divergent term in the second cumulant that exactly cancels the aforementioned divergence, $(\mathrm{C} 2)$ or (C3), in the third cumulant. In fact, this counterterm removes all divergences caused by a single occurrence of $G_{i j}^{11}$ in the entire cumulant expansion, but it is beyond the scope of this article to prove this. Also note that adding this counterterm to the Hamiltonian is equivalent to redefining the dipole polarizability $C_{1}$ as

$$
C_{1} \rightarrow C_{1}\left[1+\frac{C_{1}}{2 \sigma} \nabla^{2} G(0)\right] .
$$


This was a simple example that exhibits an induced dipole charge that produces a divergence due to interacting with itself once. Considering other multipole orders will introduce new counterterms involving different divergences, such as $\nabla^{4} G(0)$. Removing divergences due to one charge interacting with itself more than once is achieved by counterterms of higher orders in the divergences, such as $\left[\nabla^{2} G(0)\right]^{2}$. Regarding these counterterms as restoring the polarizabilities to their (divergent) bare values $C_{\text {bare }}^{(\ell)}$ from the renormalized $C^{(\ell)}$, one can write

$$
C_{\text {bare }}^{(\ell)}=C^{(\ell)}+\sum_{n=1}^{\infty} b_{n}^{(\ell)}\left[\frac{C^{(\ell)}}{\sigma} \nabla^{2 \ell} G(0)\right]^{n},
$$

where the coefficients $b_{n}^{(\ell)}$ are pure numbers appropriately chosen to cancel the offending diagrams. In this Appendix, for instance, we have explicitly found out that $b_{1}^{(1)}=1 / 2$.

\section{APPENDIX D: INDUCED MONOPOLES}

In our treatment we avoided induced monopole terms in the effective theory, i.e., terms of the form

$$
\frac{1}{2} \sum_{a} C_{a}^{(0)} h^{2}\left(\boldsymbol{r}_{a}\right),
$$

since the field of these charges - each proportional to $\log \mid \boldsymbol{r}$ $\boldsymbol{r}_{a} \mid$-violates square integrability of its gradient in $\mathbb{R}^{2}$. Such terms would describe particles with frozen vertical fluctuations. We will discuss a possible work-around for this issue.

Consider the regularized free-surface Hamiltonian

$$
\mathcal{H}[h]=\frac{1}{2} \sigma \int d^{2} r\left(h_{i}^{2}+\lambda^{-2} h^{2}\right),
$$

which approaches the tension Hamiltonian (2) in the limit of large $\lambda$. For an interface between two fluids subject to gravity (horizontal in its unperturbed state), $\lambda$ is the capillary length and is given by $\lambda=\sqrt{\sigma / g \rho}$, where $g$ and $\rho$ are the gravitational acceleration and mass density difference between the fluids, respectively. The addition of the "mass" term damps correlations over distances larger than $\lambda$, hence regularizing the infrared divergence of monopole fields. The Green function for this choice of surface energy is

$$
G\left(\boldsymbol{r}, \boldsymbol{r}^{\prime}\right)=\frac{1}{2 \pi} K_{0}\left(\left|\boldsymbol{r}-\boldsymbol{r}^{\prime}\right| / \lambda\right),
$$

instead of $(-1 / 2 \pi) \log \left|\boldsymbol{r}-\boldsymbol{r}^{\prime}\right|$, where $K_{0}(x)$ is a modified Bessel function of the second kind. After this regularization, we can safely consider particles that are completely pinned.

The effective theory of such particles involves the same induced charges as before, Eq. (15), with the addition of the monopole $(\ell=0)$ terms (D1). The reader could object that, with the new choice of kernel, there may be new terms such as $h_{i i}^{2}$ or $h_{i j k k} h_{i j}$, etc., but these terms can be eliminated using the equation of motion, $\left(-\nabla^{2}+\lambda^{-2}\right) h=0$ (for a proof, see e.g., Ref. [20]). The matching of the polarizability coefficients is done similarly by comparing the response of the induced charges to backgrounds of the form $h^{\mathrm{bg}}=\alpha r^{\ell} \cos \ell \varphi$ in the full and effective theories. One finds

$$
\delta h^{\text {full }}(\boldsymbol{r})=-\frac{R^{\ell}}{K_{\ell}(R / \lambda)} K_{\ell}(r / \lambda) \cos \ell \varphi
$$

and

$$
\delta h^{\mathrm{eff}}(\boldsymbol{r})=-\frac{C^{\ell}}{\sigma} \frac{\ell !}{2 \pi \lambda^{\ell}} K_{\ell}(r / \lambda) \cos \ell \varphi,
$$

respectively, yielding

$$
C^{(\ell)}=\frac{2 \pi R^{\ell} \lambda^{\ell} \sigma}{\ell ! K_{\ell}(R / \lambda)} .
$$

Expanding the Bessel functions, one observes that for $\ell>0$ these polarizabilities converge to those in Eq. (14) as $\lambda \rightarrow \infty$, whereas the massless limit of the monopole polarizability is

$$
C^{(0)} \rightarrow-\frac{2 \pi \sigma}{\log \left(\frac{\mathrm{e}^{\gamma} R}{2 \lambda}\right)} \quad \text { as } \lambda \rightarrow \infty,
$$

where $\gamma$ is the Euler-Mascheroni constant.

We observe that the monopole polarizability vanishes like $1 / \log (R / \lambda)$ in the massless limit of infinite capillary length that we eventually want to take. This means diagrams involving monopoles could vanish as well, unless every factor of $1 / \log (R / \lambda)$ due to a monopole polarizability is canceled by a similar factor in the numerator. Factors of this form indeed exist: They come from monopole-monopole links in the propagator product, since in the massless limit $G(r)=-(1 / 2 \pi) \log \left(\mathrm{e}^{\gamma} r / 2 \lambda\right)$. Due to the closed topology of the diagrams, there are enough monopole-monopole links to balance the vanishing monopole polarizabilities only when there are no higher-order multipoles in the diagram; replacing one monopole polarizability in the diagram costs two monopole-monopole links. In other words, the only monopole interactions that do not vanish in the massless limit are those with other induced monopoles and nothing else. This elucidates and generalizes the findings of Lehle and Oettel that monopole-dipole and monopole-quadrupole interactions indeed vanish [8].

We can now write the pair interaction between two pinned particles, on a capillary surface for which the capillary length tends to infinity. The interaction will consist of Eq. (23) due to induced multipoles of order $\ell>0$ and, based on the discussion of the previous paragraph, a part $\beta F^{\text {mon }}$ that is due solely to monopole polarizabilities. Observe that in the latter, propagators do not carry any derivatives, and therefore all the cumulants are of the same order in the interparticle separation. Hence, all cumulants must be summed for the monopole interactions. To evaluate this, one can refer to Eq. (22), keeping in mind that there is no sum over multipole orders $\ell_{i}$ now; only the monopoles are taken. Assuming identical particle radii $R$ to declutter expressions, one finds

$$
\begin{aligned}
\beta F^{\mathrm{mon}} & =-\frac{1}{2} \sum_{s=1}^{\infty} \frac{1}{s}\left[\frac{C^{(0)} G(r)}{\sigma}\right]^{2 s} \\
& =-\frac{1}{2} \sum_{s=1}^{\infty} \frac{1}{s}\left[\frac{K_{0}(r / \lambda)}{K_{0}(R / \lambda)}\right]^{2 s} .
\end{aligned}
$$


Owing to the damping of the regularized theory, this series converges for all $r(\ll \lambda \rightarrow \infty)$ to

$$
\begin{aligned}
\beta F^{\text {mon }}= & \frac{1}{2} \log \left[1-\frac{K_{0}^{2}(r / \lambda)}{K_{0}^{2}(R / \lambda)}\right] \\
= & \frac{1}{2} \log \left[K_{0}(R / \lambda)-K_{0}(r / \lambda)\right] \\
& +\frac{1}{2} \log \left[K_{0}(R / \lambda)+K_{0}(r / \lambda)\right]+\text { const. }
\end{aligned}
$$

When the massless limit $\lambda \rightarrow \infty$ is taken, the first term on the right-hand side of the last equality gives

$$
\beta F^{\text {mon }}=\frac{1}{2} \log \log \frac{r}{R},
$$

in agreement with Ref. [8]. The second term is proportional to $\log \log \left(\lambda^{2} / R r\right)$, which is associated with a force $\sim 1 / r \log \left(R r / \lambda^{2}\right) \rightarrow 0$ as $\lambda \rightarrow \infty$. We note that extension to particles of unequal radii results in the change $R \rightarrow \sqrt{R_{1} R_{2}}$ in Eq. (D10).
[1] H. B. Casimir, Proc. K. Ned. Akad. Wetensch. 51, 793 (1948).

[2] M. Bordag, U. Mohideen, and V. M. Mostepanenko, Phys. Rep. 353, 1 (2001).

[3] K. A. Milton, The Casimir Effect: Physical Manifestations of Zero-Point Energy (World Scientific, Singapore, 2001).

[4] M. Krech, Casimir Effect in Critical Systems (World Scientific, Singapore, 1994).

[5] M. Kardar and R. Golestanian, Rev. Mod. Phys. 71, 1233 (1999).

[6] A. Gambassi, J. Phys.: Conf. Ser. 161, 012037 (2009).

[7] H. Lehle, M. Oettel, and S. Dietrich, Europhys. Lett. 75, 174 (2006).

[8] H. Lehle and M. Oettel, Phys. Rev. E 75, 011602 (2007).

[9] E. Noruzifar and M. Oettel, Phys. Rev. E 79, 051401 (2009).

[10] H. Li and M. Kardar, Phys. Rev. Lett. 67, 3275 (1991).

[11] R. Golestanian, M. Goulian, and M. Kardar, Europhys. Lett. 33, 241 (1996).

[12] R. Golestanian, M. Goulian, and M. Kardar, Phys. Rev. E 54, 6725 (1996).

[13] C. Yolcu, I. Z. Rothstein, and M. Deserno, Europhys. Lett. 96, 20003 (2011).

[14] T. Emig, N. Graham, R. L. Jaffe, and M. Kardar, Phys. Rev. D 77, 025005 (2008).

[15] M. Bordag, Phys. Rev. D 73, 125018 (2006).

[16] A. Bulgac, P. Magierski, and A. Wirzba, Phys. Rev. D 73, 025007 (2006).

[17] K. A. Milton and J. Wagner, Phys. Rev. D 77, 045005 (2008).

[18] T. Emig, N. Graham, R. L. Jaffe, and M. Kardar, Phys. Rev. Lett. 99, 170403 (2007).

[19] H.-K. Lin, R. Zandi, U. Mohideen, and L. P. Pryadko, Phys. Rev. Lett. 107, 228104 (2011).
[20] I. Z. Rothstein, e-print arXiv:hep-ph/0308266v2.

[21] W. D. Goldberger and I. Z. Rothstein, Phys. Rev. D 73, 104029 (2006).

[22] R. A. Porto, A. Ross, and I. Z. Rothstein, JCAP 03 (2011) 009.

[23] C. R. Galley, A. K. Leibovich, and I. Z. Rothstein, Phys. Rev. Lett. 105, 094802 (2010).

[24] P. G. Dommersnes and J.-B. Fournier, Europhys. Lett. 46, 256 (1999).

[25] R. R. Netz, J. Phys. I (France) 7, 833 (1997).

[26] V. I. Marchenko and C. Misbah, Eur. Phys. J. E 8, 477 (2002).

[27] S. A. Safran, Statistical Thermodynamics of Surfaces, Interfaces, and Membranes (Perseus, Cambridge, 1994).

[28] M. Goulian, R. Bruinsma, and P. Pincus, Europhys. Lett. 22, 145 (1993); 23, 155(E) (1993).

[29] J.-M. Park and T. C. Lubensky, J. Phys. I (France) 7, 1217 (1996).

[30] J. L. Cardy, in Phase Transitions and Critical Phenomena, Vol. 11, edited by C. Domb and J. L. Lebowitz (Academic, New York, 1987), p. 55.

[31] T. W. Burkhardt and E. Eisenriegler, Phys. Rev. Lett. 74, 3189 (1995).

[32] B. V. Derjaguin, Kolloid-Z. 69, 155 (1935).

[33] I. Z. Rothstein, e-print arXiv:1111.0533v1.

[34] D. Stamou, C. Duschl, and D. Johannsmann, Phys. Rev. E 62, 5263 (2000).

[35] C. Yolcu and M. Deserno (in preparation).

[36] J. Riordan, Combinatorial Identities (Wiley, New York, 1968), p. 141.

[37] M. Abramowitz and I. A. Stegun, editors, Handbook of Mathematical Functions (Dover, New York, 1974). 